\title{
Critérios de seleção de patologias para investigação em Saúde
}

\author{
Pathology selection criteria for research in Health \\ Criterios de selección de patología para la investigación en Salud
}

Pedro Silvério Marques ${ }^{1}$

\section{Resumo}

Procuramos identificar os critérios que conduzem à seleção para investigação de determinadas patologias. O objetivo é verificar se tais critérios são adequados às necessidades de investigação em saúde e respondem aos problemas globais de saúde pública. Tentámos confrontar o que diz a informação científica publicada com a nossa experiência na Comissão de Ética para a Investigação Clínica. Na maioria dos repositórios consultados, há poucas referências a prioridades ou seleção de prioridades, reportando-se a aspetos concretos de patologias específicas. O motor de busca EuropePMC identifica perto de 40 mil entradas, sendo o mais significativo que encontrámos. Analisámos alguns dos artigos pela abordagem ética, metodológica para a investigação médica e suas prioridades, nomeadamente, a participação dos pacientes. A interação entre medicamentos antirretrovirais e o consumo de drogas injetáveis e a inclusão de pacientes utilizadores de injetáveis (UDI) em ensaios clínicos só foram investigadas pelas companhias farmacêuticas (e pelos próprios investigadores), pelo confronto e pressão dos principais Community Advisory Boards (CAB), nomeadamente o European CAB. A seleção e arbitragem entre patologias para a investigação não parecem despertar muito interesse por parte de investigadores e da Academia. São os financiadores da investigação, entidades, públicas ou privadas, com ou sem fins lucrativos, que selecionam as áreas a investigar. Nas empresas farmacêuticas, é o retorno económico: doenças de países e regiões de baixos recursos ou raras não têm um mercado. Têm sido as organizações multilaterais e fundações de âmbito global, com os seus financiamentos, a definir as prioridades das investigações em áreas de maior impacto global e social.

\section{Palavras-chave}

Prioridades de Pesquisa. Saúde Global. Indústria Farmacêutica.

\begin{abstract}
We try to identify the criteria leading to the selection for investigation of certain pathologies. Its goal is to verify whether these criteria are adequate to the global health research needs and respond to the main global public health problems. We tried to confront what the published scientific information says with our own experience as a member of the Ethics Committee for Clinical Research. Most of the open access repositories consulted, refers, mostly, to specific pathologies, with few references to priorities or selection of priorities. The EuropePMC search engine, the most significant of which we have found, identifies nearly 40.000 entries. We analyzed some of the articles by the ethical, methodological approach to medical research and its priorities, namely, patient participation. Interactions between antiretroviral drugs and injecting drug use and the inclusion of drug user patients in clinical
\end{abstract}

\footnotetext{
${ }^{1}$ Licenciado em Gestão; reformado, foi membro da Comissão de Ética para a Investigação Clínica, Lisboa, Portugal, e do European Community Advisory Board. https://orcid.org/0000-0001-6802-615X. E-mail: pmosmarques@gmail.com
} 
trials where only be investigated by pharmaceutical companies (and the researchers themselves), with the confrontation and pressure from the main Community Advisory Boards $(\mathrm{CAB})$, in particular European $\mathrm{CAB}$. The selection and arbitration between pathologies for research does not seem, comparatively, to raise interest on the part of researchers and the Academy. The research funders define the financeable areas. In pharmaceutical companies, it is the economic return, with little interest and social impact of the research results. Diseases in low-resource or rare countries and regions are not an interesting market. Multilateral organizations and global foundations have been responsible for prioritizing funding for research in areas of greatest global and social impact.

\section{Keywords}

Research Priorities. Global Health. Drug Industry.

\section{Resumen}

Buscamos identificar los criterios conducentes a la selección para la investigación de determinadas patologías. Su objetivo último es verificar si dichos criterios son adecuados a las necesidades de investigación en salud y a los problemas de salud pública global. Intentamos confrontar lo que dice la información científica publicada con nuestra experiencia en el Comité de Ética para la Investigación Clínica. La mayoría de los repositorios de acceso abierto consultados se refieren a patologías concretas, contienen pocas referencias a prioridades o selección de prioridades. El motor de búsqueda EuropePMC, lo más significativo que hemos encontrado, identifica cerca de 40.000 entradas. Analizamos algunos de los artículos por el enfoque ético, metodológico de la investigación médica y sus prioridades, a saber, la participación de los pacientes. Las interacciones entre los medicamentos antirretrovirales y el uso de drogas inyectables y la inclusión de pacientes UDI en ensayos clínicos solo fueron investigadas por lo enfrentamiento y presión de los principales Community Advisory Boards (CAB), en particular European CAB. La selección y arbitraje entre patologías para la investigación no parece despertar mucho interés por parte de los investigadores y de la Academia. Los financiadores de investigación definen las áreas financiables. En las empresas farmacéuticas es el retorno económico, con poco interés e impacto social de los resultados de la investigación. Los países y regiones de recursos escasos o raros no tienen interés económico, no tienen mercado. Las organizaciones y fundaciones multilaterales a nivel global se han encargado de priorizar la financiación de la investigación en áreas de mayor impacto global y social.

\section{Palabras clave}

Prioridades de Investigación. Salud Global. Industria Farmacéutica.

Encontramo-nos, em geral, perante o facto, contraditório, de, apesar de nunca se ter feito tanta e tão vasta investigação em saúde, vermos as organizações globais de saúde continuarem a lançar apelos a mais investigação em áreas e patologias que continuam a custar um pesado tributo em termos de saúde e de qualidade de vida e, portanto, de condições económicas e sociais de partes significativas da população e em diversas áreas regionais.

A primeira dificuldade na avaliação deste tema é o desconhecimento da quantidade de investigação efetuada. Na verdade, só temos conhecimento da investigação publicada 
ou, no máximo, da investigação registada. Pese embora os inúmeros apelos a que seja publicada toda a investigação realizada, incluindo solicitações e iniciativas para a partilha de dados utilizados nas diversas investigações, estes têm sido ignorados e apenas temos acesso aos resultados da investigação publicados, após escrutínio inter-pares.

Aceitando este indicador sub-rogado, será através da consulta e pesquisa dos diversos sites, de acesso livre, que publicam resultados das investigações em saúde que, de algum modo, poderemos medir e analisar a frequência de seleção de determinadas áreas de investigação e tentar explicar os possíveis critérios de determinação de prioridades. Consultámos o site da Associação Nacional de Médicos de Saúde Pública² (ANMSP), cujos Grupos de Trabalho têm como objetivo a criação de documentos técnicos, de boas práticas e baseados em evidência. As áreas dos grupos de trabalho são Sistemas de Informação em Saúde Pública; Processo de Contratualização dos Cuidados de Saúde Primários (CSP); Vacinação e Prevenção e Controlo do Tabagismo. Não consta do site qualquer repositório de publicações.

Pesquisámos, na Revista Portuguesa de Medicina Geral e Familiar (RPMGF), todos os artigos com o descritor investigação médica ${ }^{3}$. Os 41 artigos apresentados referem-se a investigações específicas e/ou a estudos de casos relacionados com patologias específicas e não apresentam qualquer reflexão ou análise referente aos critérios de investigação e/ou à seleção de prioridades. O descritor prioridades para a investigação médica não identificou qualquer artigo.

Analisando a base do Health and Human Services (HHS) de artigos científicos Medline - com o descritor setting priotities for clinical research ${ }^{4}$, encontramos 3.729 entradas, todas elas referidas a investigações específicas e/ou a estudos de casos relacionados com patologias específicas. Nos casos da malária, VIH e COVID-19, a porcentagem de trabalhos publicados referente ao estabelecimento de prioridades para investigação em relação ao total de publicações foi de, respetivamente, 0,096\%, 0,37\% e $0,056 \%$.

Por sua vez a EuropePMC ${ }^{5}$, para o mesmo descritor, apresenta 39.462 entradas. Na impossibilidade de as consultar todas, analisámos as primeiras 500 apresentadas, normalmente por ordem de contactos ou consultas, constatando que, excluindo as que

\footnotetext{
2 https://www.anmsp.pt/

3 https://www.rpmgf.pt

${ }^{4}$ https://pubmed.ncbi.nlm.nih.gov/

5 https://europepmc.org
} 
referentes a patologias, sintomatologias, regiões ou países específicos, retivemos 23 artigos referentes a metodologias para determinação de prioridades ${ }^{6}$. Alguns destes artigos serão analisados no final deste trabalho.

Há muito que as prioridades da investigação básica e clínica estão condicionadas e orientadas pelos financiamentos disponíveis ou disponibilizados. Os elevados custos de investigação e as exigências éticas e metodológicas aplicáveis, não são favoráveis à investigação de autor isolado e com recursos limitados.

É a imersão num ambiente científico favorável e incentivador da investigação que facilita a abordagem multidisciplinar e a fertilização cruzada de ideias, estudos e investigações. A investigação clínica organiza-se e desenvolve-se, assim, em torno das prioridades determinadas e financiadas por entidades, públicas ou privadas, com ou sem fins lucrativos.

Os Ministérios da Ciência, Tecnologia, Inovação ou/e Educação e as suas dotações financeiras para a investigação, Laboratórios Nacionais e Universidades, no processo de criação de bolsas e seleção de bolseiros e de atribuição de prémios de investigação, selecionam de facto as áreas em que a investigação se vai desenvolver (e as prioridades, de facto, da investigação). Os critérios de seleção são a qualidade dos projetos submetidos pelos investigadores, a sua adequação e o seu interesse para os fins da instituição e os critérios internos de afetação de fundos. O mesmo se verifica com as Fundações e Institutos que têm como missão o desenvolvimento das ciências médicas.

Mas estas áreas não parecem ser um todo coordenado de prioridades, nem nacional, nem regional, nem globalmente. Apenas as organizações multilaterais procuram consensualizar e estabelecer, como prioridades para a investigação nas ciências médicas, as patologias de maior impacto global, as que mais afetam os países e zonas mais pobres e desfavorecidas do planeta.

As empresas farmacêuticas, nomeadamente as Big Pharma, definem prioridades para as investigações realizadas nos seus laboratórios, essencialmente, com base nos potenciais

\footnotetext{
${ }^{6}$ https://europepmc.org/article/PMC/PMC7898357;https://europepmc.org/article/PMC/PMC7900798;https://europepmc.org/ article/MED/33591566;https://europepmc.org/article/MED/33589565;https://europepmc.org/article/MED/33588878; https://e uropepmc.org/article/MED/33607988;https://europepmc.org/article/PMC/PMC7898357;https://europepmc.org/article/MED/ 33577622\#free-full-text;https://europepmc.org/article/MED/33577622\#free-full-

text;https://europepmc.org/article/MED/33573701;https://europepmc.org/article/MED/33571206; https://europepmc.org/artic le/MED/33568101;https://europepmc.org/article/MED/33563626;https://europepmc.org/article/MED/33552472;https://euro pepmc.org/article/MED/33549068;https://europepmc.org/article/MED/33542106;https://europepmc.org/article/MED/335257 46;https://europepmc.org/article/MED/33517912;https://europepmc.org/article/MED/33514570;https://europepmc.org/articl e/MED/33502486;https://europepmc.org/article/MED/33499854;https://europepmc.org/article/MED/33480827;https://europ epmc.org/article/MED/33472647
} 
benefícios financeiros. O alvo dos seus produtos e das investigações que realizam é o mercado dos países ricos, com populações envelhecidas, onde a procura de soluções para combater ou adiar o envelhecimento dominam. É sintomática a comparação entre os números de trabalhos científicos sobre as patologias associadas ao envelhecimento (cardiovasculares, respiratórios, demência e degenerescência do sistema nervoso) e os dedicados, por exemplo, à malária, tripanossomíase africana humana e tantas outras doenças tropicais.

Apenas o recurso a incentivos estatais, regionais ou multilaterais, quer por subsídios diretos e transferências de tecnologias, quer por benefícios comerciais ou fiscais, tem combatido esta situação e direcionado a investigação para necessidades urgentes, crónicas ou emergentes de saúde pública.

As pressões bilaterais ocidentais, nomeadamente dos Estados Unidos, sobre os países candidatos a membros da Organização Mundial do Comércio (OMC), condicionando a sua adesão à existência de legislação sobre os direitos de propriedade intelectual, traduziuse num aumento, uniforme, dos prazos de proteção das patentes e da facilidade da sua extensão (1). A aprovação do Trade-Related Aspects of Intellectual Property Rights (TRIPS) institucionalizou a dependência da investigação médica nos países membros das prioridades e recursos das grandes farmacêuticas ocidentais e da complacência dos reguladores ocidentais.

Como exemplo significativo, os obstáculos legais, jurisdicionais, económicos e políticos levantados à produção de medicamentos anti-VIH pelo Brasil, Índia, Rússia e China. As grandes multinacionais farmacêuticas, com forte apoio norte-americano e europeu, desenvolveram inúmeros esforços, diplomáticos, económicos, institucionais e políticos para impedir o recurso, legal e previsto nas regras da OMC de recurso à quebra ou libertação de patentes, perante uma emergência de saúde pública.

$\mathrm{Na}$ década em curso, em que, em teoria, vão caducar as patentes iniciais da maior parte dos medicamentos de grande eficácia introduzidos nos finais dos anos 90 e a primeira década de 2000, vai ser interessante analisar as formas que as farmacêuticas vão encontrar para evitar a queda das patentes no domínio público.

Outro exemplo, atual, é o da oposição regulamentar e política às vacinas anti COVID19 de origem não ocidental. É o caso das vacinas chinesas, russas, indianas, que, à data em que este texto foi escrito, ainda não tinham autorização de acesso e comercialização na União Europeia. Mesmo face à manifesta incapacidade da indústria ocidental de produzir e 
fornecer a tempo as quantidades de vacinas, as dúvidas, legítimas, que são levantadas ao rigor, reprodutibilidade e transparência das investigações que levaram ao seu desenvolvimento não contribuem nem incentivam o seu esclarecimento, relevando às vezes de preconceitos e discriminação subjacentes.

Uma área relacionada é a da publicação e do acesso aos dados de base de repositórios de ensaios clínicos realizados, bem ou mal-sucedidos, e dos dados pessoais neles recolhidos que, levantando questões éticas por resolver, apresentam grande potencial para abrir caminhos novos para a investigação, evitar insistências e repetições inúteis e já exploradas.

Um dos documentos já assinalados (2) é um protocolo para a criação de um repositório de dados individuais dos participantes em ensaios clínicos controlados realizados em lares e instituições para a terceira idade e o seu alargamento para um repositório de dados para usos futuros mais alargados, atendendo à necessidade de dados pessoais de alta qualidade desta população vulnerável.

Os problemas éticos na criação destes repositórios de dados pessoais são basicamente a garantia da confidencialidade dos dados pessoais e a disponibilização e a utilização de dados recolhidos com consentimento informado para um fim específico e conhecido, para fins diferentes e indeterminados.

Adicionalmente, o reconhecimento do direito de propriedade intelectual de quem fornece ao repositório os dados inicialmente recolhidos, com implicações académicas e, eventualmente, económicas, também dificulta a criação destes repositórios de uma forma que seja rigorosa, transparente e fiscalizável.

Mas eles existem, pela apropriação privada e para utilização comercial dos dados pessoais dos participantes em ensaios e estudos desenvolvidos pela indústria e mesmo por particulares. É conhecido de todos o caso da base de dados pessoais da população islandesa, que já por diversas vezes mudou de proprietário $(3,4)$. A saga islandesa da deCODE Genetics está bem documentada através de inúmeros artigos, quer críticos quer encomiásticos, acessíveis através de qualquer motor de pesquisa. A deCODE Genetics foi estabelecida no Delaware, EUA, por um cidadão sueco, em 1996, para explorar as características genéticas únicas da população da Islândia, associadas aos registos genealógicos e aos dados de saúde. Criada legislação favorável no Parlamento islandês, foi concedido à deCODE Genetics um exclusivo de dez anos para uso e exploração desses dados. O projeto inicial nunca foi desenvolvido, em parte por intervenção do poder judicial, 
mas a empresa afirma ter os dados de saúde de 170 mil islandeses, analisado ou caracterizado o genótipo de 80 mil e sequenciado o genoma de 1.300 a 10 mil islandeses e deter informação médica e genética detalhada de 500 mil pessoas em todo o mundo. Da análise destas bases de dados surgiram desenvolvimentos promissores para a terapia genética de problemas como a doença de Alzheimer, diabetes tipo 2, doenças cardiovasculares e esquizofrenia, entre outras.

Financeiramente, a vida da deCODE nunca terá sido fácil. A promoção e venda das suas ações ao público islandês, antes da oferta pública, resultou em avultados prejuízos para os incautos subscritores: as suas ações valem hoje 3\% do preço de subscrição e um número significativo de postos de trabalho foi eliminado. A reação à oferta da Hoffmann-La Roche de 200 milhões de dólares pelo direito ao desenvolvimento de medicamentos a partir dos dados recolhidos e a recolher levou, por sentença do tribunal, à perda do acesso garantido e privilegiado aos dados de saúde da população. Em 2009, a empresa recorreu à legislação de proteção por bancarrota, continuando a recolha e análise de dados. Em 2012, a Amgen pagou 415 milhões de dólares pela empresa e criou, menos de um ano depois, por cisão do seu braço tecnológico - sistemas de análise e bases de dados, a NextCODE Health que, por montante não identificado, passou para a posse de um consórcio de investimento, a Saga Investments LLC, que inclui, entre outros, a Polaris Venture Partners e a $A R C H$ Venture Partners. A NextCODE Health foi comprada, em 2015, por 65 milhões de dólares, pela WuXi PharmaTech.

Mais recentemente temos o caso DeepMind-Royal, analisado num excelente artigo de Julia Powles e Hal Hodson (5). Resumidamente, a DeepMindTechnologies Limited, uma subsidiária do conglomerado Google, Alphabet Inc., anunciou, em 2016, o seu maior projeto na área da saúde em colaboração com o hospital Royal Free London (RFL) e o NHS Foundation Trust, para desenvolver sistemas e algoritmos de apoio clínico à gestão de doença aguda renal.

À revelia do Gabinete do Comissário para a Informação (ICO, sigla em inglês) responsável pelo cumprimento do Data Protection Act e ignorando o governance framework da Autoridade para a Investigação em Saúde (HRA, sigla em inglês), bem como as orientações do Grupo de Aconselhamento sobre Confidencialidade (CAD, sigla em inglês) sobre a divulgação de informação de saúde na ausência de consentimento explícito, o RFL transferiu milhões de dados de pacientes para a DeepMind. Só sete meses após a assinatura do acordo, cinco depois da transferência dos dados e do início do desenvolvimento e teste 
do projeto e dois anos após o anúncio público do mesmo pelos seus promotores, uma investigação jornalística independente revelou os contornos do negócio, desencadeando-se a polémica sobre a natureza, extensão e limites do acordo de transferência de dados. Os termos deste acordo parecem permitir à DeepMind construir sistemas que tenham por alvo qualquer doença em qualquer parte do corpo e não só a gestão e o tratamento direto da doença aguda renal, não existindo qualquer restrição explícita à utilização de Inteligência Artificial.

À data da publicação do artigo referido (5) - março de 2017 - desconhece-se o que é que a Google e a DeepMind estão a fazer com os dados dos utentes do NHS e qual o grau e significado do controlo que o Royal Free tem sobre o que estão a fazer. Não existe qualquer fiscalização independente nem quaisquer restrições legal ou contratualmente estabelecidas que garantam quais e como os dados serão utilizados. O montante de dados transferidos é abusivo face aos objetivos do projeto apresentado, e aqueles foram transferidos sem informação ou pedido de consentimento às pessoas identificadas nem consulta às entidades relevantes. Continua a não existir qualquer comentário ou apreciação da transferência de dados por parte dos reguladores.

Os contributos das instituições nacionais de saúde pública podem ser analisados em alguns dos artigos mencionados $(6,7,8,9)$. Esses contributos, quer nas métricas de avaliação, na investigação de prioridades, alocação de financiamentos e avaliação das práticas seguidas, servem o objetivo de maior rigor, transparência e fiscalização na utilização de fundos coletivos.

Embora sem nenhum artigo encontrado, o mesmo se deve dizer para a utilização de fundos de organizações multilaterais para investigação e ciência, normalmente por convites públicos e abertos de apresentação de candidaturas competitivas nas áreas e prioridades definidas pela organização.

A participação cidadã é abordada em cinco dos artigos selecionados $(10,11,12,13$ e 14). Qualquer que seja a metodologia de análise utilizada, existe uma diferença clara e significativa nas prioridades de investigação entre investigadores e cidadãos interessados na investigação. A qualidade de vida, quer dos sujeitos dos ensaios, quer dos pacientes que serão submetidos aos tratamentos eventualmente resultantes, nunca aparece nas prioridades definidas pelos investigadores, quando está em primeiro lugar das prioridades dos cidadãos. Quando a priorização de objetivos é alcançada consensualmente, as prioridades e a sua ordenação tornam-se diferentes. 
A participação cidadã tem sido um dos mantras mais repetidos na análise e organização dos sistemas de saúde e das instituições na área da saúde. Penso que poucas Entidades Reguladoras, Hospitais ou Centros de Saúde não terão, plasmados na sua Missão, a centralidade do cidadão e o requisito da sua colaboração nos processos de decisão. Na prática é uma declaração vazia de qualquer repercussão, no máximo podem traduzir-se na inclusão de cidadãos não profissionais de medicina em conselhos consultivos que servem, uma vez por ano, para tomar conhecimento e confirmar decisões já tomadas.

Quando as prioridades não estão consensualizadas, como já referimos, tem sido por força das organizações da sociedade civil que entidades governamentais e empresas farmacêuticas têm incluído, quando incluem, as prioridades dos cidadãos na investigação.

Os resultados não são lineares. No início da pandemia pelo VIH, a exigência pública de novos, e rapidamente aprovados, medicamentos, fez descurar a sua eficácia. Vários medicamentos, aprovados e comercializados, foram depois, rapidamente, descontinuados da prática clínica e terapêutica.

Mas uma das populações mais afetadas pelo $\mathrm{VIH}$, os utilizadores de drogas por via endovenosa - em Portugal chegaram a representar mais de 60\% dos novos casos -, fora a ação dos Community Advisory Boards (CAB), nomeadamente o European CAB (ECAB), não teria sido objeto das investigações específicas sobre as interações entre medicamentos antirretrovirais e o consumo de drogas nem incluídos em ensaios clínicos.

Como conclusão, a capacidade e a eficácia dos sistemas de saúde e das empresas farmacêuticas em desenvolver e disseminar investigação relevante para a saúde pública depende não apenas das suas capacidades científicas, tecnológicas e humanas, mas também e sobretudo, da proximidade das suas prioridades às dos financiadores e utentes ou beneficiários da investigação a realizar e da inclusão destes, de forma significativa, nos processos de decisão sobre investigação.

\section{Referências}

1. Barbieri JC, Chamas CI. O Acordo sobre Direitos de Propriedade Intelectual Relacionados ao Comércio (TRIPs) e as Políticas Públicas de Saúde e de Defesa da Biodiversidade. Revista Eletrônica de Administração. Enero-abril, 2008;(14)1:25-49.

2. Irvine L, Burton JK, Ali M, Quinn TJ, Goodman C. Protocol for the development of a repository of individual participant data from randomised controlled trials conducted in adult care homes (the Virtual International Care Homes Trials Archive (VICHTA)). Trials. 2021; 22(157). Disponível em: https://doi.org/10.1186/s13063-021-05107-w 
3. Chadwick RF, Shickle D, Ten Have HA, Wiesing U (Eds.). The Ethics of Genetic Screening. Springer, Dordrecht; 1999. 256 p.

4. Michael D. Lemonick. The Iceland Experiment. Time Magazine. Sunday, Feb. 12, 2006. Disponível em: http://content.time.com/time/magazine/article/0,9171,1158968,00.html

5. Powles J, Hodson H. Google DeepMind and healthcare in an age of algorithms. Health Technol (Berl). 2017;7(4):351-367. doi: 10.1007/s12553-017-0179-1. Epub 2017 Mar 16. PMID: 29308344; PMCID: PMC5741783.

6. Guthrie S, Cochrane G, Deshpande A, Macaluso B, Larivière V. Understanding the contribution of UK public health research to clinical guidelines: a bibliometric analysis. F1000Res. 2019 Jul 15;8:1093. doi: 10.12688/f1000research.18757.1. PMID: 33552472; PMCID: PMC7845157.

7. Tatham KC, McAuley DF, Borthwick M, Henderson NG, Bashevoy G, Brett SJ. The National Institute for Health Research Critical Care Research Priority Setting Survey 2018. J Intensive Care Soc. 2020 Aug;21(3):198-201. doi: 10.1177/1751143719862244. Epub 2019 Jul 8. PMID: 32782458; PMCID: PMC7401440.

8. Ballreich JM, Gross CP, Powe NR, Anderson GF. Allocation of National Institutes of Health Funding by Disease Category in 2008 and 2019. JAMA Netw Open. 2021 Jan 4;4(1):e2034890. doi: 10.1001/jamanetworkopen.2020.34890. PMID: 33502486; PMCID: PMC7841468.

9. Seixas BV, Regier DA, Bryan S, Mitton C. Describing practices of priority setting and resource allocation in publicly funded health care systems of high-income countries. BMC Health Serv Res. 2021 Jan 27;21(1):90. doi: 10.1186/s12913-021-06078-z. PMID: 33499854; PMCID: PMC7839200.

10. Chamberlain SA, Estabrooks CA, Keefe JM, Hoben M, Berendonk C, Corbett K, Gruneir A. Citizen and stakeholder led priority setting for long-term care research: identifying research priorities within the Translating Research in Elder Care (TREC) Program. Res Involv Engagem. 2020 May 15;6:24. doi: 10.1186/s40900-020-00199-1. PMID: 32467774; PMCID: PMC7229578.

11. Parker L, Grundy Q, Fabbri A, Mintzes B, Bero L. 'Lines in the sand': an Australian qualitative study of patient group practices to promote independence from pharmaceutical industry funders. BMJ Open. 2021 Feb 9;11(2):e045140. doi: 10.1136/bmjopen-2020045140. PMID: 33563626; PMCID: PMC7875302.

12. Crowe S, Fenton M, Hall M, Cowan K, Chalmers I. Patients', clinicians' and the research communities' priorities for treatment research: there is an important mismatch. Res Involv Engagem. 2015 Jun 25;1:2. doi: 10.1186/s40900-015-0003-x. Erratum in: Res Involv Engagem. 2015 Dec 23;1:14. PMID: 29062491; PMCID: PMC5598091. 
13. Ludwig, C, Graham ID, Lavoie J et al. Ethical considerations for engaging frail and seriously ill patients as partners in research: sub-analysis of a systematic review. Res Involv Engagem. 2021;7(8). Disponível em: https://doi.org/10.1186/s40900-021-00254-5

14. Deane KH, Flaherty H, Daley DJ, Pascoe R, Penhale B, Clarke CE, Sackley C, Storey $\mathrm{S}$. Priority setting partnership to identify the top 10 research priorities for the management of Parkinson's disease. BMJ Open. 2014 Dec 14;4(12):e006434. doi: 10.1136/bmjopen2014-006434. PMID: 25500772; PMCID: PMC4281559.

\section{Como citar este artigo}

Silvério Marques P. Critérios de seleção de patologias para investigação em Saúde. Cadernos Ibero-Americanos de Direito Sanitário. 2021 jul./set.;10(3):18-28

https://doi.org/10.17566/ciads.v10i3.784 